\title{
C-Reactive Protein +1444 C/T Polymorphism Is Associated with the Susceptibility to Pulmonary Tuberculosis
}

\author{
Yuzhong Xu, ${ }^{1}$ Minggang Cheng $\mathbb{D},{ }^{1}$ and Xiong Wang $\mathbb{D}^{2}$ \\ ${ }^{1}$ Department of Clinical Laboratory, Shenzhen Baoan Hospital, Southern Medical University, Shenzhen 518000, China \\ ${ }^{2}$ Department of Laboratory Medicine, Tongji Hospital, Tongji Medical College, Huazhong University of Science, \\ Wuhan 430030, China \\ Correspondence should be addressed to Minggang Cheng; cmg70@163.com and Xiong Wang; wangxiong@tjh.tjmu.edu.cn
}

Received 3 October 2020; Revised 22 November 2020; Accepted 14 December 2020; Published 21 December 2020

Academic Editor: Praveen Bharti

Copyright (C) 2020 Yuzhong Xu et al. This is an open access article distributed under the Creative Commons Attribution License, which permits unrestricted use, distribution, and reproduction in any medium, provided the original work is properly cited.

Objective. The $\mathrm{T}$ allele of $\mathrm{C}$-reactive protein $(\mathrm{CRP})+1444 \mathrm{C} / \mathrm{T}$ ( $\mathrm{rs} 1130864)$ polymorphism was associated with increased risk for some inflammatory conditions. The objective of the study was to explore the association between the CRP $+1444 \mathrm{C} / \mathrm{T}$ polymorphism with the susceptibility to pulmonary tuberculosis (PTB) in a Chinese population. Methods. This case-control study enrolled $480 \mathrm{PTB}$ patients and 480 healthy controls. The CRP $+1444 \mathrm{C} / \mathrm{T}$ polymorphism was determined using Sanger sequencing. The odds ratio (OR) and 95\% confidence interval (CI) were assessed to examine the strength of genetic correlation. Results. The genotype and allele frequencies of PTB patients differed from controls (CT vs. CC, OR =1.924, 95\% CI: $1.099-3.371$, adjusted $P$ value $=0.022$; T vs. C, $\mathrm{OR}=1.884,95 \%$ CI: $1.085-3.273$, adjusted $P$ value $=0.024)$. Stratified analysis by sex found that PTB patients' genotype and allele frequencies differed from controls in the male subgroup but not the female subgroup. Conclusion. In conclusion, the minor $\mathrm{T}$ allele of $\mathrm{CRP}+1444 \mathrm{C} / \mathrm{T}$ polymorphism was associated with increased PTB risk.

\section{Introduction}

Tuberculosis (TB) is an infectious disease caused by $M y c o-$ bacterium tuberculosis (Mtb), which ranks among the top 10 causes of death worldwide. According to the global tuberculosis report 2019 from WHO (https://www.who .int/tb/publications/global_report/en/), about 10.0 million people fell ill with TB in 2018, and 1.7 billion people were latent TB infection (LTBI). Early and accurate diagnosis of TB is critical for achieving the goal of global tuberculosis control [1]. Most of the Mtb infections are LTBI, which is asymptomatic. When the host immune system weakens, the Mtb becomes active and causes active TB with clinical symptoms. The development of TB is affected by the characteristics of $\mathrm{Mtb}$, the host immune responses, and the genetic susceptibility [2].

Plasma C-reactive protein (CRP) is an acute-phase reactant produced by hepatocytes after an IL-6 stimulus and plays an essential role in chronic and acute inflammation. Plasma CRP is a useful nonspecific biomarker for the assessment of disease activity in inflammatory conditions, including TB. Serum CRP was increased in TB, being highest in advanced disease. This level fell with the antiTB drug treatment and also correlated with the clinical response $[3,4]$. The CRP $+1444 \mathrm{C} / \mathrm{T}$ (rs1130864) polymorphism was located in the $3^{\prime}$ untranslated region $\left(3^{\prime}\right.$-UTR) of the CRP gene. The $\mathrm{T}$ allele carriers had higher CRP serum concentrations [5]. This polymorphism has been found to be associated with the susceptibility to several inflammatory conditions, such as systemic lupus erythematosus (SLE) and cardiovascular disease (CVD) $[6,7]$. However, the association between the CRP $+1444 \mathrm{C} / \mathrm{T}$ (rs1130864) polymorphism and the susceptibility to pulmonary TB (PTB) is not yet studied.

The objective of the study was to explore the association between the CRP $+1444 \mathrm{C} / \mathrm{T}$ polymorphism and the 
susceptibility to PTB in a Chinese population including 480 PTB patients and 480 healthy controls.

\section{Materials and Methods}

2.1. Subjects. 480 PTB patients were enrolled from Shenzhen Baoan Hospital, Southern Medical University from January 2018 to January 2020. PTB was diagnosed by chest X-ray radiography and clinical symptoms including (fever, hemoptysis, weight loss, dyspnea, chest pain, and night sweats), combined with positive result of culture, Mtb-specific PCR, T-SPOT.TB assay, or Xpert MTB/RIF. Patients with HIV infection, immunodeficiency disease, diabetes mellitus, tumor, or other lung diseases were excluded. This study was approved by the ethics committee of Shenzhen Baoan Hospital. Informed consent was obtained from all subjects.

2.2. Genotyping of the CRP $+1444 C / T$ Polymorphism. $200 \mu \mathrm{l}$ peripheral blood was collected, and genomic DNA was extracted using the GeneRotex 96 Nucleic Acid Extraction workstation (TIANLONG, Xi'an, China). The following primers were used: forward, $5^{\prime}$-ATATTAATAAGGAGCT CGTTAACTATGCTGGGACA- ${ }^{\prime}$ and reverse, $5^{\prime}$-TTCT CAGCTCTTGCCTTATGAG-3' according to a previous study [8]. The PCR fragments were sequenced using ABI 3500 Dx (ABI, San Diego, CA, USA), and the results were blasted on NCBI.

2.3. Statistical Analyses. All data were presented as mean \pm SD and analyzed using SPSS 18.0 (SPSS Inc., Chicago, Illinois, USA). Differences of age between two groups were analyzed using unpaired Student's $t$-test. Chi-squared $\left(\chi^{2}\right)$ test was applied to assess the Hardy-Weinberg equilibrium (HWE) in controls. Odds ratios (OR) and 95\% confidence intervals (CIs) were calculated using binary logistic regression analysis, adjusted by age combined with/without sex. $P$ value $<0.05$ was considered statistically significant.

\section{Results}

3.1. Demographic and Clinical Characteristics of Subjects. 480 PTB patients (mean age: $39.81 \pm 12.65$ years) including 330 males and 150 females and matched 480 healthy controls (mean age: $39.88 \pm 14.61$ years) including 330 males and 150 females were enrolled. No significant difference was found in either age or male ratio between cases and controls. Detailed clinical characteristics were listed in Table 1.

3.2. Association between $C R P+1444 C / T$ Polymorphism and the Susceptibility to PTB. The controls followed the HWE principle $(P=0.641)$, suggesting representative control subjects. The genotype distribution of CRP $+1444 \mathrm{C} / \mathrm{T}$ polymorphism in cases was significantly different from controls (CT vs. $\mathrm{CC}, \mathrm{OR}=1.924,95 \% \mathrm{CI}: 1.099-3.371$, adjusted $P$ value $=$ $0.022)$. Stratified analysis by sex found that the genotype distribution of CRP $+1444 \mathrm{C} / \mathrm{T}$ polymorphism in cases was significantly different from male controls (CT vs. CC, OR = 2.178, 95\% CI: 1.127-4.208, adjusted $P$ value $=0.021)$ but not females (Table 2).
TABLE 1: Characteristics of the study population.

\begin{tabular}{lccc}
\hline Variable & Case & Control & $P$ value \\
\hline $\begin{array}{l}\text { Number } \\
\text { Sex }\end{array}$ & 480 & 480 & \\
$\quad$ Male & $330(68.75 \%)$ & 330 & \\
$\quad$ Female & $150(31.25 \%)$ & 150 & \\
Age & $39.81 \pm 12.65$ & $39.88 \pm 14.61$ & 0.938 \\
$\quad$ Male & $40.22 \pm 12.48$ & $40.32 \pm 15.12$ & 0.926 \\
$\quad$ Female & $38.93 \pm 13.03$ & $38.93 \pm 13.41$ & 1.000 \\
\hline
\end{tabular}

The allele frequencies of CRP $+1444 \mathrm{C} / \mathrm{T}$ polymorphism was significantly different from controls ( $\mathrm{T}$ vs. $\mathrm{C}, \mathrm{OR}=$ 1.884, 95\% CI: $1.085-3.273$, adjusted $P$ value $=0.024)$. Stratified analysis by sex found that the genotype distribution of CRP $+1444 \mathrm{C} / \mathrm{T}$ polymorphism in cases was significantly different from male controls ( $\mathrm{T}$ vs. $\mathrm{C}, \mathrm{OR}=2.120,95 \% \mathrm{CI}$ : 1.109-4.052, adjusted $P$ value $=0.023)$ but not females (Table 3).

\section{Discussion}

Detection of TB is still challenging, although great advances have been achieved in new diagnostic methods [1]. Difficulty exists in detecting several cases such as acid-fast cacillus (AFB) smear-negative sputum, adolescent TB, and reactivation of LTBI. The diagnosis of adolescent TB is challenging as adolescent TB is paucibacillary with nonspecific symptoms. In the early stage, up to $50 \%$ of adolescent $\mathrm{TB}$ can be asymptomatic [9]. Inexpensive and simple diagnostic methods have not been found yet. Several biomarkers have been identified for diagnosis and differentiation between active TB and LTBI $[10,11]$.

CRP is an acute-phase reactant with a short half-life and has been used as one of the most frequent ways of monitoring inflammation [12]. CRP participates in the immune response by binding to its main ligand phosphorylcholine or intracellular ligands, such as chromatin, ribonucleoprotein, and histones on dying or dead cells, activates the complement pathway and Fc-receptor-mediated effectors pathways, and promotes phagocytosis [13-15]. CRP, soluble intercellular adhesion molecule-1 (sICAM1), procalcitonin, and neopterin are serum biomarkers which exhibit macrophage activation in TB [4]. Serum CRP level was increased in TB patients, especially in those patients with AFB smearpositive sputum. Serum CRP level was correlated with severe lung tissue damage [16]. CRP level decreased to a normal level after anti-TB treatment, indicating an effective therapeutic response [4].

The gene coding for CRP is located at 1q.23.2. Some functional polymorphisms within the CRP gene have been found to affect serum CRP levels, including the CRP $+1444 \mathrm{C} / \mathrm{T}$ (rs1130864) polymorphism, rs3091244, rs3093077, and rs11265265 [5, 17-20]. Associations between the CRP $+1444 \mathrm{C} / \mathrm{T}$ polymorphism and the susceptibility to several diseases have been widely studied. The CRP $+1444 \mathrm{C} / \mathrm{T}$ polymorphism was associated with SLE risk, and patients carrying 
TABLE 2: Genotype distribution of CRP $+1444 \mathrm{C} / \mathrm{T}$ polymorphism in all subjects.

\begin{tabular}{lcccc}
\hline Number & Case & Control & OR & Adjusted $P$ value \\
\hline Genotype & 480 & 480 & & \\
CC & $443(92.29 \%)$ & $460(95.83 \%)$ & $1.924(1.099-3.371)$ & 0.022 \\
CT & $37(7.71 \%)$ & $20(4.17 \%)$ & 1 & 0.021 \\
Male number & 330 & 330 & $2.178(1.127-4.208)$ \\
CC & $301(91.21 \%)$ & $316(95.76 \%)$ & & 1 \\
CT & $29(8.79 \%)$ & $14(4.24 \%)$ & $1.352(0.457-3.999)$ & 0.585 \\
Female number & 150 & 140 & & \\
CC & $142(94.67 \%)$ & $6(4 \%)$ & & \\
CT & $8(5.33 \%)$ & & & \\
\hline
\end{tabular}

The $P$ value was adjusted by age combined with/without sex.

TABLE 3: The allele frequencies of CRP $+1444 \mathrm{C} / \mathrm{T}$ polymorphism in all subjects.

\begin{tabular}{lccccc}
\hline Model & Allele & Case & Control & OR (95\% CI) & Adjusted $P$ value* $^{*}$ \\
\hline \multirow{2}{*}{ Overall } & $\mathrm{C}$ & $923(96.15 \%)$ & $940(97.92 \%)$ & 1 & 0.024 \\
& $\mathrm{~T}$ & $37(3.85 \%)$ & $20(2.08 \%)$ & $1.884(1.085-3.273)$ & 1 \\
\multirow{2}{*}{ Male } & $\mathrm{C}$ & $631(95.61 \%)$ & $646(97.88 \%)$ & $2.120(1.109-4.052)$ & 0.023 \\
& $\mathrm{~T}$ & $29(4.39 \%)$ & $14(2.12 \%)$ & 1 & 0.590 \\
\multirow{2}{*}{ Female } & $\mathrm{C}$ & $292(97.33 \%)$ & $294(98 \%)$ & $1.343(0.460-3.918)$ & \\
& $\mathrm{T}$ & $8(2.67 \%)$ & $6(2 \%)$ & & \\
\hline
\end{tabular}

The $P$ value was adjusted by age combined with/without sex.

the $\mathrm{T}$ allele presented higher CRP levels [6]. The CRP $+1444 \mathrm{C} / \mathrm{T}$ polymorphism was significantly associated with increased posttraumatic stress disorder (PTSD) symptoms and increased CRP level [21]. The minor allele of CRP $+1444 \mathrm{C} / \mathrm{T}$ polymorphism was associated with decreased risk of depression in women aged at least 65 years. CRP gene variant was also associated with serum levels in a gender-specific manner. The CRP $+1444 \mathrm{C} / \mathrm{T}$ polymorphism may influence circulating CRP level [22]. In the current study, we found that the $\mathrm{T}$ allele of CRP $+1444 \mathrm{C} / \mathrm{T}$ polymorphism was associated with increased PTB risk, especially in males. In the included 480 PTB patients, only 150 patients were female. The limited sample size of female may be the reason why this association was not found in female.

Some limitations exist in our study. First, the sample size was small, and only 150 female cases were enrolled. A larger sample size in female population deserves further study of the association between CRP $+1444 \mathrm{C} / \mathrm{T}$ polymorphism and $\mathrm{PTB}$ risk in female. Second, the information of the serum CRP level was lacking. Third, the $P$ value was only adjusted by age and/or sex.

In conclusion, our data indicate that the minor $\mathrm{T}$ allele of CRP $+1444 \mathrm{C} / \mathrm{T}$ polymorphism was associated with increased PTB risk in a Chinese population, especially in males.

\section{Data Availability}

The datasets used and analyzed during the current study are available from the corresponding author on reasonable request.

\section{Ethical Approval}

The Ethics Committee of Shenzhen Baoan Hospital approved this study.

\section{Consent}

Participants provided written informed consent before the commencement of this study. Participant consent was provided for the publishing of this article.

\section{Conflicts of Interest}

The authors have no competing interests to declare.

\section{Authors' Contributions}

Yuzhong Xu collected samples. Minggang Cheng designed the experiment. Xiong Wang analyzed and interpreted the data. All authors read and approved the final manuscript.

\section{References}

[1] G. Walzl, R. Mcnerney, N. Du Plessis et al., "Tuberculosis: advances and challenges in development of new diagnostics and biomarkers," The Lancet. Infectious diseases, vol. 18, no. 7, pp. e199-e210, 2018.

[2] L. Abel, J. El-Baghdadi, A. A. Bousfiha, J. L. Casanova, and E. Schurr, "Human genetics of tuberculosis: a long and winding road," Philosophical transactions of the Royal Society of London. Series B, Biological sciences, vol. 369, 2014. 
[3] B. Kashyap, N. Gupta, P. Dewan, P. Hyanki, and N. P. Singh, "High sensitivity $\mathrm{C}$ reactive protein: an adjunct diagnosis in ruling out Pediatric tuberculosis," Indian journal of clinical biochemistry: IJCB, vol. 35, no. 2, pp. 211-217, 2020.

[4] S. Soedarsono and M. C. Subiantoro, "Changes of CRP serum levels in pulmonary TB patients with AFB smear-positive sputum before and two months after receiving anti-tuberculosis drug treatment," The Indian Journal of Tuberculosis, vol. 66, no. 1, pp. 134-138, 2019.

[5] D. J. Brull, N. Serrano, F. Zito et al., "Human CRP gene polymorphism influences CRP Levels," Arteriosclerosis, Thrombosis, and Vascular Biology, vol. 23, no. 11, pp. 2063-2069, 2003.

[6] F. Delongui, M. A. B. Lozovoy, T. M. V. Iriyoda et al., "C-reac-

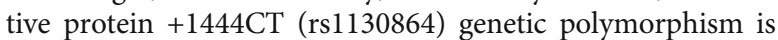
associated with the susceptibility to systemic lupus erythematosus and C-reactive protein levels," Clinical Rheumatology, vol. 36, no. 8, pp. 1779-1788, 2017.

[7] S. Schulz, H. Ludike, M. Lierath et al., "C-reactive protein levels and genetic variants of CRP as prognostic markers for combined cardiovascular endpoint (cardiovascular death, death from stroke, myocardial infarction, and stroke/TIA)," Cytokine, vol. 88, pp. 71-76, 2016.

[8] S. Willot, S. Vermeire, M. Ohresser et al., "No association between $\mathrm{C}$-reactive protein gene polymorphisms and decrease of C-reactive protein serum concentration after infliximab treatment in Crohn's disease," Pharmacogenetics and Genomics, vol. 16, no. 1, pp. 37-42, 2006.

[9] R. F. Carvalho, A. C. C. Carvalho, L. G. C. Velarde et al., "Diagnosis of pulmonary tuberculosis in children and adolescents: comparison of two versions of the Brazilian Ministry of Health scoring system," Revista do Instituto de Medicina Tropical de Sao Paulo, vol. 62, 2020.

[10] Z. Y. Chen, L. Wang, L. Gu et al., "Decreased expression of CD69 on T cells in tuberculosis infection resisters," Frontiers in Microbiology, vol. 11, p. 1901, 2020.

[11] Y. Luo, Y. Xue, Q. Lin et al., “A combination of iron metabolism indexes and tuberculosis-specific antigen/phytohemagglutinin ratio for distinguishing active tuberculosis from latent tuberculosis infection," International journal of infectious diseases : IJID : official publication of the International Society for Infectious Diseases, vol. 97, pp. 190-196, 2020.

[12] M. B. Pepys and G. M. Hirschfield, "C-reactive protein: a critical update," The Journal of Clinical Investigation, vol. 111, no. 12, pp. 1805-1812, 2003.

[13] T. W. Du Clos, "C-reactive protein reacts with the U1 small nuclear ribonucleoprotein," Journal of Immunology, vol. 143, pp. 2553-2559, 1989.

[14] T. W. Du Clos, L. T. Zlock, and R. L. Rubin, "Analysis of the binding of C-reactive protein to histones and chromatin," Journal of Immunology, vol. 141, pp. 4266-4270, 1988.

[15] L. Marnell, C. Mold, and T. W. Du Clos, "C-reactive protein: ligands, receptors and role in inflammation," Clinical Immunology, vol. 117, no. 2, pp. 104-111, 2005.

[16] A. E. Shapiro, T. Hong, S. Govere et al., "C-reactive protein as a screening test for HIV-associated pulmonary tuberculosis prior to antiretroviral therapy in South Africa," AIDS, vol. 32, no. 13, pp. 1811-1820, 2018.

[17] C. S. Carlson, S. F. Aldred, P. K. Lee et al., "Polymorphisms within the C-reactive protein (CRP) promoter region are associated with plasma CRP levels," American Journal of Human Genetics, vol. 77, no. 1, pp. 64-77, 2005.
[18] Y. C. Huang, C. C. Chen, T. Y. Wang et al., "C-reactive protein gene variants and their serum levels in early adult-onset type 2 diabetes mellitus," In Vivo, vol. 33, no. 5, pp. 1685-1690, 2019.

[19] S. Mukherjee and A. Tripathi, "Association of serum Creactive protein level and polymorphisms with susceptibility to dengue infection and severe clinical outcome among eastern Indian patients," Medical Microbiology and Immunology, vol. 209, no. 5, pp. 631-640, 2020.

[20] P. Navarro, O. De Dios, T. Gavela-Perez, L. Soriano-Guillen, and C. Garces, "Relationship between polymorphisms in the CRP, LEP and LEPR genes and high sensitivity C-reactive protein levels in Spanish children," Clinical Chemistry and Laboratory Medicine, vol. 55, no. 11, pp. 1690-1695, 2017.

[21] V. Michopoulos, A. O. Rothbaum, T. Jovanovic et al., "Association of CRP genetic variation and CRP level with elevated PTSD symptoms and physiological responses in a civilian population with high levels of trauma," The American Journal of Psychiatry, vol. 172, no. 4, pp. 353-362, 2015.

[22] M. L. Ancelin, A. Farre, I. Carriere, K. Ritchie, I. Chaudieu, and J. Ryan, "C-reactive protein gene variants: independent association with late-life depression and circulating protein levels," Translational Psychiatry, vol. 5, no. 1, p. e499, 2015. 